\title{
Cationic Hofmeister Series of Wettability Alteration in Mica-Water- Alkane Systems
}

\author{
B. Bera, ${ }^{\dagger}$ N. Kumar, ${ }^{\dagger}$ M. H. G. Duits, M. A. Cohen Stuart, and F. Mugele* \\ Physics of Complex Fluids Group, University of Twente, Enschede 7522 NB, The Netherlands \\ Supporting Information
}

ABSTRACT: The specific interaction of ions with macromolecules and solid-liquid interfaces is of crucial importance to many processes in biochemistry, colloid science, and engineering, as first pointed out by Hofmeister in the context of (de)stabilization of protein solutions. Here, we use contact angle goniometry to demonstrate that the macroscopic contact angle of aqueous chloride salt solutions on mica immersed in ambient alkane increases from near-zero to values exceeding $10^{\circ}$, depending on the type and concentration of cations and $\mathrm{pH}$. Our observations result in a series of increasing ability of cations to induce partial wetting in the order $\mathrm{Na}^{+}, \mathrm{K}^{+}<\mathrm{Li}^{+}<$ $\mathrm{Rb}^{+}<\mathrm{Cs}^{+}<\mathrm{Ca}^{2+}<\mathrm{Mg}^{2+}<\mathrm{Ba}^{2+}$. Complementary atomic

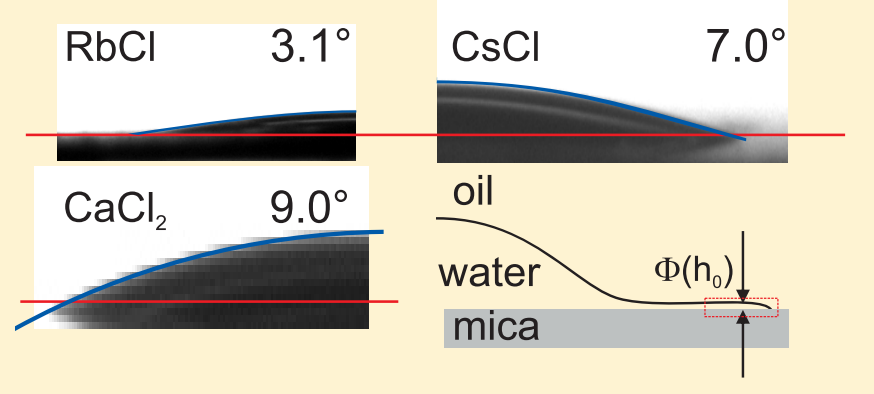
force microscopy measurements show that the transition to partial wetting is accompanied by cation adsorption to the micaelectrolyte interface, which leads to charge reversal in the case of divalent cations. In addition to electrostatics, hydration forces seem to play an important role, in particular for the monovalent cations.

\section{INTRODUCTION}

Competitive wetting of oil and water on solid surfaces plays an important role in many applications of colloid and interface science, including environmental two-phase flows, remediation of environmental contamination, ${ }^{1}$ Pickering emulsions, separation of emulsions, ${ }^{2}$ and the recovery of crude oil from geological reservoirs. ${ }^{3-5}$ In many of these applications, the fluids involved are rather complex due to the presence of various solutes. Changes in the fluid composition can have important consequences for the performance of the system, e.g., by affecting colloidal stability, ${ }^{6-8}$ adsorption, ${ }^{9,10}$ and resulting flow properties. ${ }^{11}$ Understanding wettability ${ }^{12}$ is particularly challenging because wetting involves the interaction of three types of interfaces, the solid-oil (so), solidwater (sw), and the oil-water (ow) interfaces that are all susceptible to variations of the composition of the fluids. Only the balance of all three tensions $\gamma_{\mathrm{so}}, \gamma_{\mathrm{sw}}$, and $\gamma$ determines the contact angle following Young's equation

$$
\gamma_{\mathrm{sw}}+\gamma \cos \theta=\gamma_{\mathrm{so}}
$$

Understanding variations of the contact angle, thus, requires simultaneous understanding of the response of all three interfacial tensions to changes of the fluid composition. Classical thermodynamics of interfaces teaches us that any solute that is either attracted to or repelled from an interface will decrease or increase the corresponding interfacial tension. ${ }^{12,13}$ While surfactants are arguably the most important common additive to control interfacial tensions and wettability, almost any other solute does the same unless a fortuitous compensation of molecular interaction forces suppresses any interfacial activity. In the present work, we focus on the effect of dissolved salts on the wettability of oilwater-mineral systems, in particular, aqueous electrolytes on mica, an aluminosilicate mineral, in an ambient alkane. While the work is motivated by the possibility of enhancing the production of crude oil by reducing the salinity of the water that is injected into geological reservoirs in the course of the standard water flooding oil recovery process, our primary goal is to identify the microscopic mechanisms controlling wettability in this idealized model system.

For both oil-water and mica-water interfaces the adsorption of ions from the aqueous phase has been studied in great detail throughout several decades. Specific attention has been paid to air-water interfaces. They serve as a general representative of hydrophobic-water interfaces also including interfaces of water with inert oils such as alkanes. In the case of air-water interfaces, the interfacial tension increases with the increasing concentration for the majority of "inert" inorganic ions. The basic origin of this trend was explained by Onsager and Samaras, ${ }^{14}$ who realized that ions dissolved in a more polar medium, water, are repelled from an interface with a less polar medium, oil, by electrostatic image forces. Even for point charges, this leads to a depletion of ions from the interface and hence to an increase of the surface tension following Gibbs' adsorption equation. Yet, dielectric polarization of the media involved is not the only force acting on the ions. The surface

Received: September 28, 2018

Revised: October 24, 2018

Published: October 24, 2018 
tension is also affected by the polarizability of the ion itself and also by hydration effects, which are highly ion-specific. For many anions, their larger size leads to a strong polarizability of the ion that overcompensates the effect of image charge forces, and thus leads to accumulation rather than depletion. In recent years, advanced experimental techniques such as X-ray spectroscopy have generated a wealth of detailed information about density profiles of various ions reflecting their specific affinity towards (or depletion from) the water surface. ${ }^{15-17}$

Mineral-water interfaces have classically been studied using titration measurements, electrokinetic techniques such as streaming potential measurements, as well as various types of surface force measurements (surface forces apparatus (SFA), atomic force microscopy (AFM), colloidal probe-AFM). ${ }^{9,10,18}$ Mica has been studied in particular detail because it can be prepared by cleaving to generate clean atomically smooth surfaces over macroscopic areas with well-defined tetrahedra of silicon oxide at the surface. Mica displays a rather strong intrinsic negative surface charge caused by isomorphic substitution of $\mathrm{Si}$ by $\mathrm{Al}$ atoms in the top layer of the crystal lattice. Cations are known to adsorb to these interfaces to compensate the negative charge density. Their affinity increases with the increasing ion size along the alkali series, as reported consistently in both electrokinetic and force measurements. Results for divalent cations are less consistent. Early SFA measurements came to the conclusion that the strong hydration of $\mathrm{Ca}^{2+}$ and $\mathrm{Mg}^{2+}$ suppress surface adsorption. ${ }^{19}$ Later, however, charge reversal of micaelectrolyte interfaces was found for divalent cation concentrations of the order of $10-20 \mathrm{mM}^{20}$ implying rather strong adsorption. This is consistent with the stronger decreases of the $\zeta$-potential as a function of divalent cations' concentration compared to monovalent ones. ${ }^{21}$ Many aspects of these measurements could be captured by surface speciation models that describe the adsorption of ions to specific sites on the surface in combination with a standard Poisson-Boltzmann description of the diffuse part of the electric double layer.

More recently, high-resolution AFM experiments ${ }^{22-26}$ as well as X-ray reflectivity measurements ${ }^{27}$ confirmed the overall increasing adhesion with the increasing alkali ion size as well as the stronger adhesion of divalent compared to monovalent cations. In addition, these experiments provided a lot more details $^{23,25,27-29}$ regarding, e.g., competing inner shell and outer shell adsorption, ${ }^{27,30}$ the lateral position of adsorption sites, $^{31}$ and in particular, the role of hydration water for the stabilization of adsorbed ions. ${ }^{23,25}$

The increasing experimental insights have been complemented by theoretical developments attempting to account for the observations in terms of ion size, charge, and polarizability, invoking Coulombic ${ }^{32,33}$ and dispersion ${ }^{34,35}$ forces, and crucially, hydration. ${ }^{36,37}$ Yet, disentangling the various individual contributions often proved very challenging and perhaps even conceptually impossible. Molecular dynamics simulations seem to provide a way out of this stalemate. Rather than attempting to identify specific interaction mechanisms, simulation results can be used to extract interaction potentials of the mean force that include the full complexity of the ionspecific behavior at interfaces. Such calculations have confirmed many of the experimental trends. In particular, they consistently highlight the prominent role of hydration ${ }^{38,39}$ for ion-specific adsorption to hydrophilic solid substrates. In this context, classical concepts such as the like-likes-like principle have received renewed attention and support. ${ }^{26,40,41}$
Given the importance of ion-specific effects for the adsorption of ions to the individual interfaces, ${ }^{42-50}$ it is plausible that they will also affect the wetting properties. Indeed, a number of studies analyzed the wettability of electrolytes on solid surfaces. ${ }^{51-53}$ Yet, these reports mainly focused on the $\mathrm{pH}$ dependence and evaluated the variation of the charge density and energy of the diffuse part of the electric double layer making use of Lippmann's equation. ${ }^{13}$ Little attention was paid to specific ion effects. Recently, some of us reported very distinct variations of the wettability of aqueous solutions of $\mathrm{CaCl}_{2}$ on mica in ambient decane as a function of concentration and $\mathrm{pH}^{54}$ While the solutions displayed immeasurably small contact angles at low salt concentrations and $\mathrm{pH}$, finite contact angles were found for concentrations exceeding a few tens of millimolar, first at elevated $\mathrm{pH}$ and for higher concentrations beyond $\approx 100 \mathrm{mM}$ across a wide $\mathrm{pH}$ range from 3 to 10 . In contrast, $\mathrm{NaCl}$ and $\mathrm{KCl}$ solutions were found to display near-zero contact angles for all concentrations and $\mathrm{pH}$ values. Complementary streaming potential measurements showed that the transition from vanishing to finite contact angles for $\mathrm{CaCl}_{2}$ coincided with the reversal of the charge of the mica-electrolyte interfaces. A numerical model based on Derjaguin-Landau-Verwey-Overbeek (DLVO) theory and surface speciation provided an excellent description of the experimental observations for the limited set of salts investigated at that time. A subsequent more detailed modeling effort highlighted the role of charge regulation and short-range chemical forces for the exact value of the contact angles. ${ }^{55}$

In the present article, we extend our previous work on salt and $\mathrm{pH}$-dependent wettability measurements to a wider range of ions, namely for the chloride salts the alkali metals $\mathrm{Li}, \mathrm{Na}, \mathrm{K}$, $\mathrm{Rb}, \mathrm{Cs}$, and for the earth alkaline metals $\mathrm{Mg}, \mathrm{Ca}$, and $\mathrm{Ba}$. Except for $\mathrm{Na}^{+}$and $\mathrm{K}^{+}$, all of these cations induce a transition to finite contact angles beyond the salt- and $\mathrm{pH}$-dependent threshold concentrations. We identified an order of increasing capability of cations to induce partial wetting, which we will refer to as "Hofmeister series of wettability" for the present system. The macroscopic contact angle measurements are complemented by surface charge measurements using atomic force microscopy (AFM). While the AFM results confirm that the reduction of electrostatic repulsion by cation adsorption indeed promotes partial wetting. Yet, in contrast to the suggestions of our previous experiments based on a limited set of cations, the present measurements also show that charge reversal is not a prerequisite for achieving finite contact angles.

The manuscript is organized as follows: in the subsequent section, we describe the experimental methods and procedures. In the Results and Discussion section, we report first the brine composition-dependent contact angle measurements followed by a description of a model of the wetting properties based on Derjaguin-Landau-Verwey-Overbeek (DLVO) theory including an unknown part due to short-range chemical (hydration) forces. Subsequently, we describe our atomic force microscopy (AFM) measurements and the resulting surface charge densities. In the Results and Discussion section, we rationalize our results and point out the importance of hydration forces.

\section{EXPERIMENTAL SECTION}

Materials. Anhydrous alkanes (decane, heptane, hexadecane: $>99 \%$ ) were purchased from Sigma-Aldrich. Prior to the experiments, they were purified by passing them five times through a column of alumina powder $\left(\mathrm{Al}_{2} \mathrm{O}_{3}\right.$, Sigma-Aldrich, Puriss grade $\left.>98 \%\right)$ to 


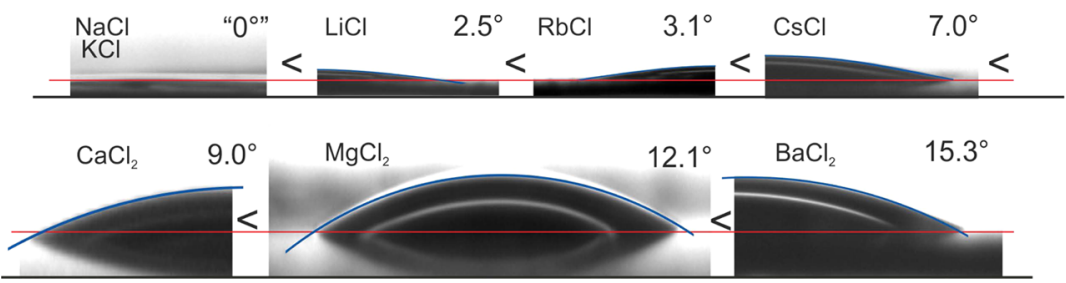

Figure 1. Snapshots of sessile brine drops on mica in ambient decane (concentration: $1 \mathrm{M} ; \mathrm{pH} \mathrm{9).} \mathrm{NaCl}$ and $\mathrm{KCl}$ drops assume near-zero, yet finite contact angles $\left(<1^{\circ}\right)$, as confirmed by visualizing the contact line in top view (ellipsometric) imaging. ${ }^{54}$ We observe the finite contact angle not only for the heavier $\mathrm{Rb}^{+}$and $\mathrm{Cs}^{+}$ions but also for $\mathrm{Li}^{+}$. Consistently higher contact angles are found for divalent cations. Blue lines: circle fitting.

remove surface-active impurities until the interfacial tension between the oil and water remained constant to within $1^{\circ}$ within $30 \mathrm{~min}$. Ultrapure water (Millipore, Synergy UV Instruments, resistivity 18 $\mathrm{M} \Omega$ ) was used to prepare solutions of the salts in a concentration range of $1 \mathrm{mM}$ to $1 \mathrm{M}$. All salts were purchased from Sigma-Aldrich (Analytical grade, purity $>99.5 \%$ ) and used without further purification. The $\mathrm{pH}$ of the solutions is adjusted to 3, 6, and 9 by adding $\mathrm{HCl}$ or $\mathrm{HNO}_{3}$ or $\mathrm{NaOH}(0.1 \mathrm{M}$, Sigma-Aldrich). For the $\mathrm{pH}$ range investigated, this implies a maximum concentration of the added ions of $10^{-5} \mathrm{M}$, which is small compared to the minimum concentration of the dominant salt of $1 \mathrm{mM}$. In practice, the $\mathrm{pH}$ of the aqueous stock solution was typically set to about $0.2 \mathrm{pH}$ units higher than the target value to compensate upfront the effect of dissolving $\mathrm{CO}_{2}$ from the ambient air atmosphere during handling. All solutions were kept in air-tight containers between their preparation and the contact angle measurements to minimize any contamination, including $\mathrm{CO}_{2}$ dissolution. Measurements of the $\mathrm{pH}$ after the contact angle experiments confirmed that the $\mathrm{pH}$ decrease over time was not more than the provided offset.

Muscovite mica sheets (B\&M Mica Company Inc.; initial thickness $340 \mu \mathrm{m})$ were cleaved in air using adhesive tape and immersed within seconds into an oil-filled cuvette for the subsequent contact angle measurements. A few test experiments with mica sheets cleaved directly under oil with a tweezer and a knife edge resulting in identical results, demonstrating that the potential contamination from the ambient air did not have any measurable influence. Oxidized silicon wafers with an amorphous silicon oxide layer (thickness: $30 \mathrm{~nm}$ ) for the calibration measurements of the AFM tips were cleaned using a combination of Piranha solution (mixture of $30 \%$ conc. $\mathrm{H}_{2} \mathrm{O}_{2}$ and $70 \%$ conc. $\mathrm{H}_{2} \mathrm{SO}_{4}$ ) followed by extensive rinsing with ultrapure water and plasma treatment for $30 \mathrm{~min}$.

Contact Angle Goniometry. Macroscopic contact angle measurements were performed using a commercial contact angle goniometer (OCA 20L, Dataphysics Inc.) using the sessile-drop method with a drop volume of $2 \pm 0.2 \mu \mathrm{L}$. Droplets were deposited by slowly approaching a syringe needle with the pendant drop to the mica surface. Upon water-mica contact, the droplets spreaded and detached from the needle. Contact angle values for each fluid composition shown in this work are averages of 25 independent measurements on at least 5 different solid substrates. Additional pendant drop measurements were performed to determine the oil/ water interfacial tension of the oil-water interface (see the Supporting Information).

Contact angles ranging from 0 to approximately $15^{\circ}$ were extracted from video snapshots using the tangent and circle-fitting data analysis software (SCA 22) provided with the instrument. The minimum absolute contact angle that can be determined on reflective surfaces is approximately $2^{\circ}$. The absolute value of the contact angle depends on details of the measurement conditions such as the exact viewing angle, drop volume, illumination settings, and the exposure time of the camera. By standardizing all these measurement conditions, we achieved a relative accuracy of $\pm 1^{\circ}$. Given the smallness of the contact angles in our systems it was not possible to determine a receding contact angle. On the basis of the deposition protocol, the measured contact angle should thus be considered as advancing contact angles. In addition to the side view imaging, we occasionally determined the contact angle $(\theta)$ by measuring the base diameter of the drop $(2 R)$ as determined from bottom view images in combination with the known drop volume $\left(V_{\text {cap }}\right)$ and the geometric relation: $V_{\text {cap }}=\frac{1}{3} \pi R^{3}\left(2-3 \sin \theta+\sin ^{3} \theta\right)$ for spherical caps. A more detailed description of the goniometry measurement procedures is provided in the Supporting Information.

Atomic Force Microscopy. Dynamic force spectroscopy measurements were performed in an amplitude modulation mode (AM-AFM) with an Asylum Research Cypher ES AFM equipped with photothermal excitation along with a sealed fluid cell. The AFM cantilever is completely immersed in the aqueous salt solution, which is sandwiched between the sample and the top surface of the cantilever holder. The measurements were performed using rectangular silicon cantilevers (MikroMasch NSC36/Cr-Au BS) with a gold backside coating. Prior to use, the cantilevers are cleaned by rinsing with an ethanol/isopropanol mixture followed by plasma cleaning in residual air gas for $20-25 \mathrm{~min}$. The cantilever spring constant $\left(k_{\mathrm{c}}\right)$, quality factor $(Q)$, and eigen frequency $\left(f_{0}\right)$ were determined by measuring the thermal noise spectrum at a tip-sample distance of approximately $150 \mathrm{~nm}$. Typical values are: $k_{\mathrm{c}} \sim 0.85 \mathrm{~N} / \mathrm{m}$, $f_{0} \sim 22 \mathrm{kHz}$, and $Q \sim 3.0$. The AFM tips were slightly blunted by bringing them into frictional contact with the solid substrate to increase the tip-sample interaction forces. This procedure resulted in typical tip radii of $20-40 \mathrm{~nm}$, as determined by high-resolution scanning electron microscopy after the measurements. Measured amplitude and phase vs tip-sample distance data were converted into forces using standard procedures. For details of these procedures, see 56.

Subsequently, the charge density of the mica surfaces is extracted from the tip-sample interaction forces by fitting the force curves to a disjoining pressure model based on DLVO theory including a charge regulation boundary condition that allows for ion adsorption. This procedure involves calibration measurements of the surface charge of the tip on an oxidized silicon wafer of identical chemical composition. Details of these procedures are described in refs 56 and 57 .

\section{RESULTS AND DISCUSSION}

Contact Angle Measurements. Figure 1 shows side view images of a series of drops for all types of salts investigated. For each of these drops, the concentration of the respective salt was set to $1 \mathrm{M}$ and the $\mathrm{pH}$ was adjusted to 9. These images summarize the essence of our observation. There is a clear trend of the contact angle increasing systematically from (in side view images) immeasurably small values for $\mathrm{NaCl}$ and $\mathrm{KCl}$ solutions to values of $\approx 15^{\circ}$ in the case of $\mathrm{BaCl}_{2}$. In the absence of any added salt, the contact angle is equally immeasurably small as in the case of $\mathrm{NaCl}$ and $\mathrm{KCl}$. Figure 1, thus, provides an order of increasing capability of the cations to increase the water contact angle. We denote this order as the cationic Hofmeister series of wettability.

$$
\mathrm{Na}^{+}, \mathrm{K}^{+}<\mathrm{Li}^{+}<\mathrm{Rb}^{+}<\mathrm{Cs}^{+}<\mathrm{Ca}^{2+}<\mathrm{Mg}^{2+}<\mathrm{Ba}^{2+}
$$



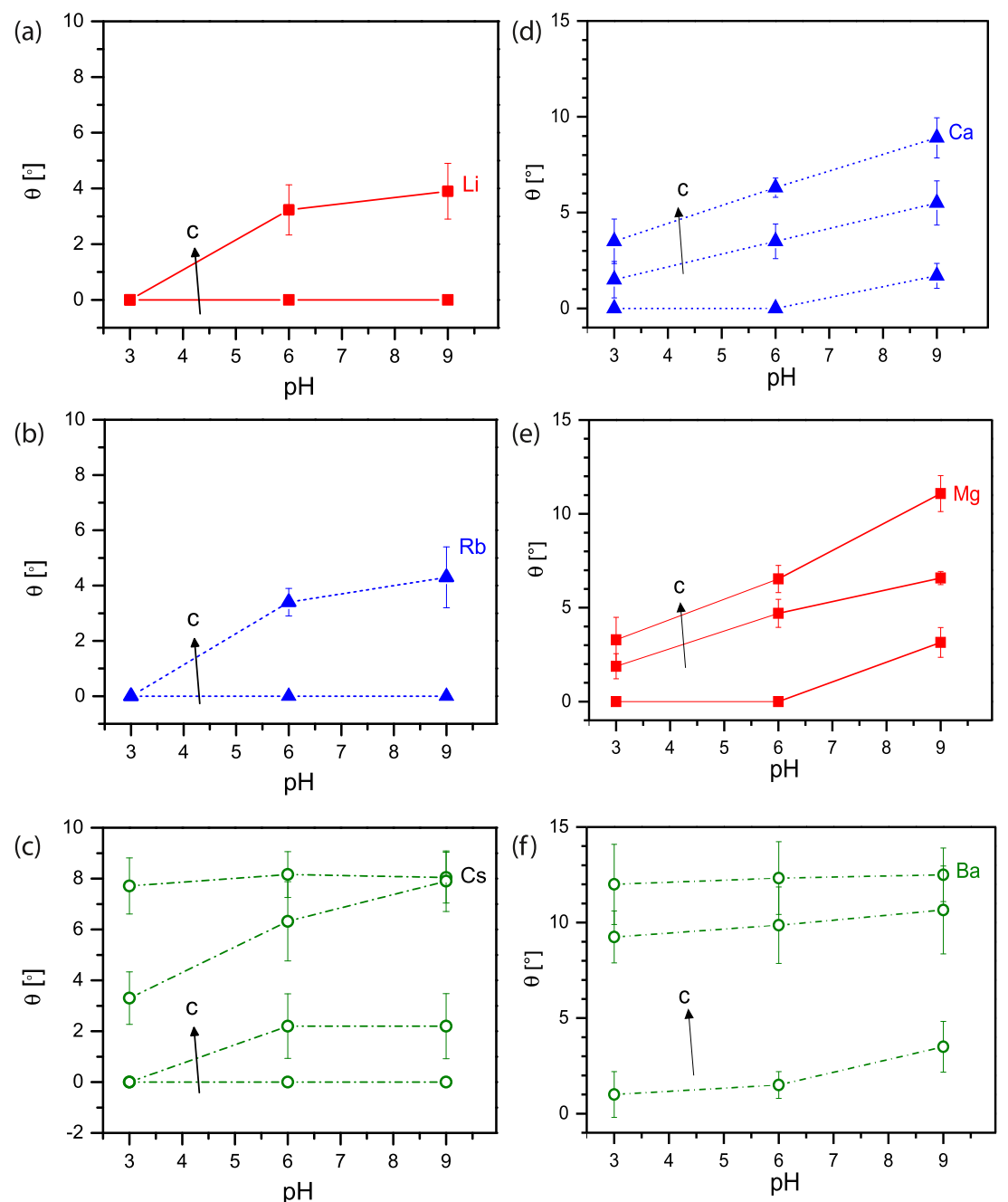

Figure 2. Equilibrium contact angle of aqueous drops on mica in ambient decane vs $\mathrm{pH}$ for various cation species and salt concentrations increasing as indicated by the arrows. Top, alkali chlorides: (a) $\mathrm{LiCl}$, at $<1$ and $1 \mathrm{M}$ conc. (b) $\mathrm{RbCl}$, at $<1$ and $1 \mathrm{M}$ conc. (c) CsCl, at $0.01,0.1$, and $1 \mathrm{M}$ conc.; $\mathrm{NaCl} / \mathrm{KCl}$ displays immeasurably small contact angles and are not shown here. Bottom, earth alkaline chlorides: $(\mathrm{d}) \mathrm{CaCl}_{2}$, at $0.05,0.1$, and $1 \mathrm{M}$ conc. (e) $\mathrm{MgCl}_{2}$, at $0.05,0.1$, and $1 \mathrm{M}$ conc. (f) $\mathrm{BaCl}_{2}$, at $0.01,0.1$, and $1 \mathrm{M}$ conc. All measurements are averaged values of 25 independent experiments; error bar: statistical standard deviation.

To provide a more complete and quantitative account of our basic observation, we conducted the same type of contact angle measurements for the full concentration range from $1 \mathrm{mM}$ to 1 $\mathrm{M}$ for $\mathrm{pH} 3$ to 9 . The results of these experiments are summarized in Figure $2 \mathrm{a}-\mathrm{c}$ for the alkali series of monovalent cations and in Figure $2 \mathrm{~d}-\mathrm{f}$ for the divalent earth alkaline cations. For $\mathrm{Na}^{+}$and $\mathrm{K}^{+}$ions, the contact angle is immeasurably small for all concentrations and $\mathrm{pH}$ values, as reported previously. ${ }^{54}$ For all other salts, a clear trend towards increasing the contact angle with increasing salt concentration and $\mathrm{pH}$ is observed. For all these salts, there is a threshold concentration beyond which the water contact angle is finite. The threshold concentration deceases with the increasing $\mathrm{pH}$. The relative order of the different cations that is shown in Figure 1 was found to persist for all other concentrations and $\mathrm{pH}$ values, supporting the robustness of this Hofmeister series of wettability. In summary, there are three key observations:

1. for all salts except for $\mathrm{NaCl}$ and $\mathrm{KCl}$, a finite macroscopic contact angle is observed beyond some threshold concentration.
2. whenever a finite contact angle can be detected, it increases upon increasing $\mathrm{pH}$ and/or the salt concentration.

3. there are strong ion-specific effects as summarized by the Hofmeister series of wettability.

A few additional remarks are in order: first of all, the divalent cations systematically display a stronger dewetting capability than the monovalent ones, i.e., the threshold concentrations for observing finite contact angles are lower and the contact angles at identical concentrations and $\mathrm{pHs}$ are higher. Secondly, within the series of alkali ions, the order of cations in eq 2 essentially corresponds to the classical "direct" Hofmeister series of protein precipitation, ${ }^{39}$ with the exception of $\mathrm{Li}^{+}$compared to the position of $\mathrm{Na}^{+}$and $\mathrm{K}^{+}$. The relative order of the divalent cations with respect to the monovalents, however, does not follow the expectations of the classical Hofmeister series, yet consistent with their stronger adsorption to the mica surface (see below). The reversal of the order of $\mathrm{Mg}^{2+}$ with respect to $\mathrm{Ca}^{2+}$ may have similar reasons as in the positioning of $\mathrm{Li}^{+}$wrt $\mathrm{Na}^{+}$and $\mathrm{K}^{+}$. (Figure SI6 in the Supporting Information shows the detailed comparison between $\mathrm{Mg}^{2+}$ and $\mathrm{Ca}^{2+}$ for a series of additional intermediate 


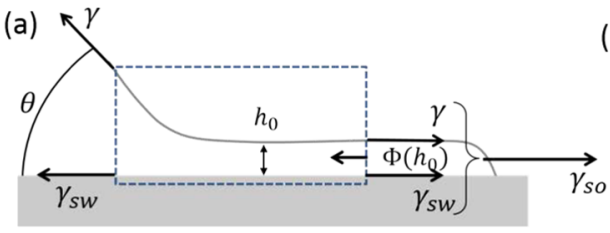

(c)

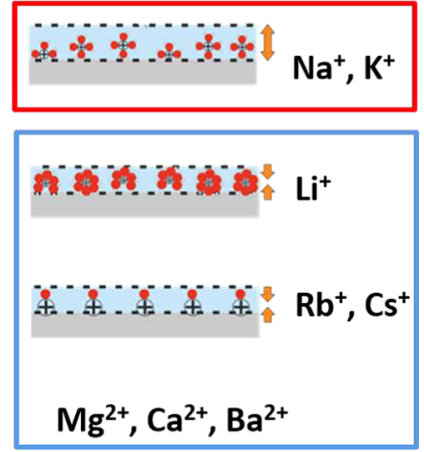

(b)

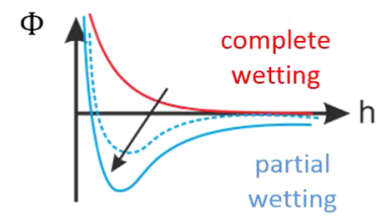

Figure 3. (a) Force balance arising from interfacial tensions leading to the contact angle $\theta$ of a drop requires the supplementary contribution of interaction potential $\Phi(h)$ when there is a molecularly thin film of equilibrium thickness $h_{0}$ present next to the drop. (b) Schematic examples of repulsive and attractive $\Phi(h)$, where negative $\Phi(h)$ with a pronounced minimum leads to a finite contact angle. The arrow depicts the gradual change of cations following our "cationic Hofmeister series". (c) Left: In the case of monovalent cations, both mica-water and oil-water interfaces remain negatively charged (above), while divalent cations above a certain concentration reverses the surface charge of mica (below). Right: Ionspecific adsorption leads to different results even among monovalent cations; a moderate sized and moderately hydrated ion: $\mathrm{Na}^{+}$or $\mathrm{K}^{+}(\mathrm{above})$ adsorb less strongly. Very small cations (middle) such as $\mathrm{Li}^{+}$get hydrated strongly and as a result adsorb significantly with their large hydrated size. Larger (bare) cations such as $\mathrm{Cs}^{+}$(bottom) adsorb strongly due to their large size despite the lack of hydration; the large size leads to lateral interaction as well as interaction with the oil-water interface. Figure (a) and (b) adapted from Mugele et al. ${ }^{54}$

$\mathrm{pH}$ values.) Thirdly, we note that the contact angle of $\mathrm{NaCl}$ and $\mathrm{KCl}$ solutions as well as pure water is actually finite, despite the appearance of the side view images. This can be revealed by recording bottom view imaging of the drops through the transparent substrate and by imaging ellipsometry measurements, as reported before ${ }^{54}$ (data not shown here). In fact, finite contact angles are expected in this system given the attractive nature of the van der Waals interaction between the oil-water and the mica-water interface, as can be estimated based on bulk refractive indices and polarizabilities of the materials. Finally, we note that additional tests with $\mathrm{Ca}\left(\mathrm{NO}_{3}\right)_{2}$ at variable $\mathrm{pH}$ and concentration displayed qualitatively the same behavior as the corresponding $\mathrm{CaCl}_{2}$ solutions, as shown in Supporting Information Figure SI7.

Macroscopic Force Balance and Disjoining Pressure. To understand our key observations, we need to relate the macroscopic contact angle to the microscopic physicochemical properties of the various interfaces involved in the wetting process. To this end, we will regard the observed contact angles as equilibrium values. Doing so, we know from Young's equation, eq 1, that the contact angle is determined by the balance of the three interfacial tension forces at the contact line. It is obvious that tensions of the oil-water and the solidwater interface can (and indeed do) change upon changing the composition of the brine, whereas, at first glance, one might think that the solid-oil interfacial tension might not depend on the brine composition. Yet, it does: Young's force balance applies on the macroscopic scale, i.e., outside the range of molecular interaction forces at the interface, as indicated by the dotted control volume in Figure 3. Systems with rather small contact angles such as the present one usually fall into the regime of "pseudopartial wetting". ${ }^{12}$ In this regime, the macroscopic drop is accompanied by a microscopic equilibrium film of the droplet phase that is interspersed between the solid and the ambient medium next to the drop, as sketched in Figure 3a. The thickness $h_{0}$ of this equilibrium film typically falls in the range between zero and at most a few nanometers. It is governed by the balance of molecular interaction forces, as given by the minimum of the effective interface potential $\Phi(h)$ (blue curves in Figure $3 b$, which describes the excess tension of the thin film caused by the proximity of the two interfaces). In our earlier work, ${ }^{54}$ we showed using imaging ellipsometry that such an equilibrium film is indeed present next to droplets of $\mathrm{NaCl}$ and $\mathrm{CaCl}_{2}$ solutions. It was found to extend for at least $1 \mathrm{~mm}$ next to the macroscopic drop and-as qualitatively expected-it displayed a thickness ranging from $\approx 0.5$ to $10 \mathrm{~nm}$, depending on the type of cation and the salt concentration. From a macroscopic perspective, the existence of this film implies that the value of $\gamma_{\text {so }}$ is in fact the equilibrium tension of the composite solid-oil interface including the microscopic water film. In the language of the effective interface potential, this means that $\gamma_{\text {so }}$ is given by the sum of $\gamma, \gamma_{\mathrm{sw}}$ and the excess tension of the equilibrium film, i.e., $\Phi\left(h_{0}\right)$, as illustrated in Figure 3a

$$
\gamma_{\mathrm{so}}=\gamma+\gamma_{\mathrm{sw}}+\Phi\left(h_{0}\right)
$$

This thin water film is in diffusive equilibrium with the bulk aqueous drop. As a consequence, $\gamma_{\text {so }}$ is also expected to depend on the composition of the brine. To understand the observed increase of the contact angle increasing salt concentrations and $\mathrm{pH}$, we therefore need to consider the variations of all three interfacial tensions.

According to the Gibbs law, the variation of the tension $\gamma_{i}$ of an arbitrary interface $i$ as a function of the fluid composition is determined by the surface excess $\Gamma$ with respect to some reference state following $\mathrm{d} \gamma=-\Gamma \mathrm{d} \mu$, where $\mu=k T \ln \left(c / c_{0}\right)$ is the chemical potential of the dissolved species with bulk concentration $c$. ( $c_{0}$ is the bulk reference concentration.) Spontaneous adsorption of solute to an interface, i.e., a positive surface excess $\Gamma$, thus implies a reduction of $\gamma_{i}$. Vice versa, a negative surface excess implies a spontaneous increase of $\gamma_{i}$. As mentioned in the introduction, hydrophobic-water interfaces including oil-water and air-water interfaces display a negative surface excess for the majority of "simple" inorganic ions such as the ones studied here because image charge forces repel electrostatic charges in the highly polarizable aqueous phase from the interface with the less polarizable ambient oil. As explained above, for hydrophobic-water interfaces the effect is dominated by anions. ${ }^{15,16}$ For air-water interfaces $\mathrm{d} \gamma / \mathrm{d} c \approx 1.5$ $\mathrm{mJ} / \mathrm{m}^{2}$ for all chloride salts of alkali metals, with a slightly 
lower coefficient for $\mathrm{Cs}^{+}$because of the higher polarizability of this large ion. $\mathrm{MgCl}_{2}$ and $\mathrm{CaCl}_{2}$ dependence is approximately twice as strong. ${ }^{43}$ We performed a few test experiments with selected salts to reproduce these trends (see Supporting Information Figure SI5). Indeed, we find positive values of $\mathrm{d} \gamma /$ $\mathrm{d} c$ with absolute values approximately half the ones mentioned above for water-air interfaces. This is consistent with the higher dielectric constant of decane compared to air. Qualitatively, an increase of $\gamma$ with the increasing salt concentration translates into an increasing contact angle. Yet, while the variation of $\gamma$ certainly contributes to the absolute values of the contact angles observed, it is unlikely that the oil-water interface is the primary driver for the observed phenomena for the following reasons. First of all, if we insert eq 3 into Young's equation, we can rewrite the force balance as

$$
\gamma(\cos \theta-1)=\Phi\left(h_{0}\right)
$$

In this equation, all quantities depend on the salt concentration and $\mathrm{pH}$. The variation of the left-hand side with fluid composition is given by $\Delta(\gamma(\cos \theta-1))=\Delta \gamma \times(\cos \theta-$ 1) $+\gamma \times \Delta \cos \theta$. Since $\theta$ varies approximately between 0 and $10^{\circ}$ in our experiments and $\gamma$ between 50 and $52 \mathrm{~mJ} / \mathrm{m}^{2}$, it is clear that the variation of $\gamma$ is less important than the variation of $\cos \theta$. Moreover, as mentioned above, ion specificity at hydrophobic-water interfaces is generally dominated by anions and not by cations. The strong cation effect in our experiments can certainly not be caused by the oil-water interface. This statement is also supported by the comparison of the contact angle data for $\mathrm{CaCl}_{2}$ and $\mathrm{Ca}\left(\mathrm{NO}_{3}\right)_{2}$ solutions: $\mathrm{d} \gamma / \mathrm{d} c$ for $\mathrm{CaCl}_{2}$ is $60 \%$ higher than for $\mathrm{Ca}\left(\mathrm{NO}_{3}\right)_{2}$ because of the larger surface affinity of the nitrate anion compared to chloride. ${ }^{45}$ Nevertheless, the contact angle data for the two different salts agree with each other within error (see Figure SI7).

Similarly, the solid-water interface cannot be at the origin of the phenomenon. As discussed in the introduction, it is wellestablished that cations spontaneously adsorb to mica-water interfaces in an ion-specific manner ${ }^{10,19,21,27,29,58,59}$ with adsorption energies ranging between a few and several tens of $\mathrm{kJ} / \mathrm{mol}$. Hence, $\gamma_{\mathrm{sw}}$ in Young's equation is expected to decrease with increasing salt concentration. This would lead to a decrease in $\theta$ with the increasing salt concentration, in contrast to the experimental findings. If we phrase the force balance equation in the form of eq $4, \gamma_{\mathrm{sw}}$ formally even completely drops out of the equation. Hence, we conclude that the observed increase in $\theta$ with the increasing salt concentration and $\mathrm{pH}$ must be driven primarily by the variation of the tension $\gamma_{\text {so }}$ of the composite solid-oil interface, or, according to eq 4 , by $\Phi\left(h_{0}\right)$. (Note that the density of adsorbed ions at the mica surface will be different in the thin equilibrium film compared to the macroscopic drop.) This difference, however, is included in $\Phi\left(h_{0}\right)$, and thus does not change the cancellation of $\gamma_{\mathrm{sw}}$ in the derivation of eq 4 .

The effective interface potential is determined by the molecular interaction forces acting across the thin water film next to the drop. ${ }^{12}$ In classical continuum wetting theory, $\Phi$ is decomposed into different contributions arising from van der Waals interaction $\Phi_{\mathrm{vdW}}$, electrostatic interactions $\Phi_{\mathrm{el}}$, and short-range chemical forces $\Phi_{\text {ch }}$ such as hydration forces

$$
\Phi(h)=\Phi_{\mathrm{vdW}}(h)+\Phi_{\mathrm{el}}(h)+\Phi_{\mathrm{ch}}(h)
$$

The functional form of the last term is generally not very wellknown because it depends on atomic scale details of the system. Frequently, it is modeled by an empirical exponentially decaying function with a decay length of the order of $1 \mathrm{~nm} .^{9,10}$ This functional form can be derived from a Landau-type free energy functional involving an order parameter of the water phase, as first proposed by Marčelja and Radic. ${ }^{60}$ DLVO theory provides expressions for the first two terms. Namely, the van der Waals contribution is given by

$$
\Phi_{\mathrm{vdW}}=A / 12 \pi h^{2}
$$

where $A=-0.4 \times 10^{-21} \mathrm{~J}$ is the Hamaker constant of the decane-water-mica system. (The exact value varies by $10-$ $20 \%$ depending on the salt species and concentration.) The negative sign of the Hamaker constant indicates that the two interfaces attract each other and that van der Waals interaction favors partial wetting.

The most interesting term for our present discussion is the electrostatic interaction $\Phi_{\mathrm{el}}(h)$. It requires a solution of the Poisson-Boltzmann equation for the distribution of ions and electrostatic potential within the thin film. ${ }^{9,10}$ Frequently, this is done using either constant (i.e., $h$-independent) charge or constant potential boundary conditions. For the present situation, however, where $h_{0}$ becomes comparable to or smaller than the Debye screening length, this is not justified and the regulation of the interfacial charge due to adsorption (or desorption) of ions needs to be taken into account. The resulting nonlinear charge regulation boundary conditions leads to a self-consistent mathematical problem that is most easily addressed numerically. To understand the physical principle leading to the observed wetting transition, it is sufficient to consider the simplified linearized version of $\Phi_{\mathrm{el}}(h)$. Here, we linearize both the Poisson-Boltzmann equation, which then reduces to the Debye-Huckel equation and the charge regulation boundary conditions. The latter leads to the linearized "constant regulation" approximation. ${ }^{55,61,62}$ The resulting expression reads

$$
\Phi_{\mathrm{el}}(h)=\frac{k_{\mathrm{B}} T}{e} \frac{2 \Psi_{\mathrm{sw}, \infty} \Psi_{\mathrm{so}, \infty} \mathrm{e}^{-\kappa h}-\left[\Delta_{\mathrm{sw}} \Psi_{\mathrm{so}, \infty}^{2}+\Delta_{\mathrm{so}} \Psi_{\mathrm{sw}, \infty}^{2}\right] \mathrm{e}^{-2 \kappa h}}{1-\Delta_{\mathrm{sw}} \Delta_{\mathrm{so}} \mathrm{e}^{-2 \kappa h}}
$$

Here, $\Psi_{\mathrm{i}, \infty}$ with $\mathrm{i}=\mathrm{sw}$, ow are the (diffuse layer) potentials of the mica-water and the oil-water interface for $h \rightarrow \infty$ and $\Delta_{i}$ are the corresponding regulation parameters of each interface. These parameters interpolate between a constant charge $\left(\Delta_{\mathrm{i}}=\right.$ $-1)$ and constant potential $\left(\Delta_{\mathrm{i}}=1\right)$ behavior. ${ }^{18,61}$ Analysis of eq 7 shows that there are four generic wetting scenarios, namely complete wetting, pseudopartial wetting, true partial wetting $\left(h_{0}=0\right)$, and a metastable wetting configuration in which a thin film can be metastable despite the existence of a true partial wetting state of lower energy, ${ }^{55}$ depending on the values of the $\Delta_{\mathrm{i}}^{\prime}$ 's and $\Psi_{\mathrm{i}}^{\prime}$ 's. For the present system, both regulation parameters are negative and the diffuse layer potentials in the absence of adsorbing ions are negative, too. In this case, $\Phi_{\mathrm{el}}$ is positive and monotonically increasing with decreasing $h$, as illustrated by the red curve in Figure $3 \mathrm{~b}$. This corresponds to a situation of complete wetting. For $\mathrm{NaCl}$ and $\mathrm{KCl}$ solutions, this situation prevails for all salt concentrations and $\mathrm{pH}$ values. In contrast, for $\mathrm{CaCl}_{2}$, the sign of the charge (and hence the potential) of the mica-water interface reverses beyond a certain $\mathrm{pH}$-dependent threshold concentration, as described in ref 20 . Under these conditions, the interface potential develops a local minimum with a finite equilibrium thickness $h_{0}$, as illustrated by the blue curves in Figure $2 \mathrm{~b}$. Consequently, the system undergoes a transition to partial 

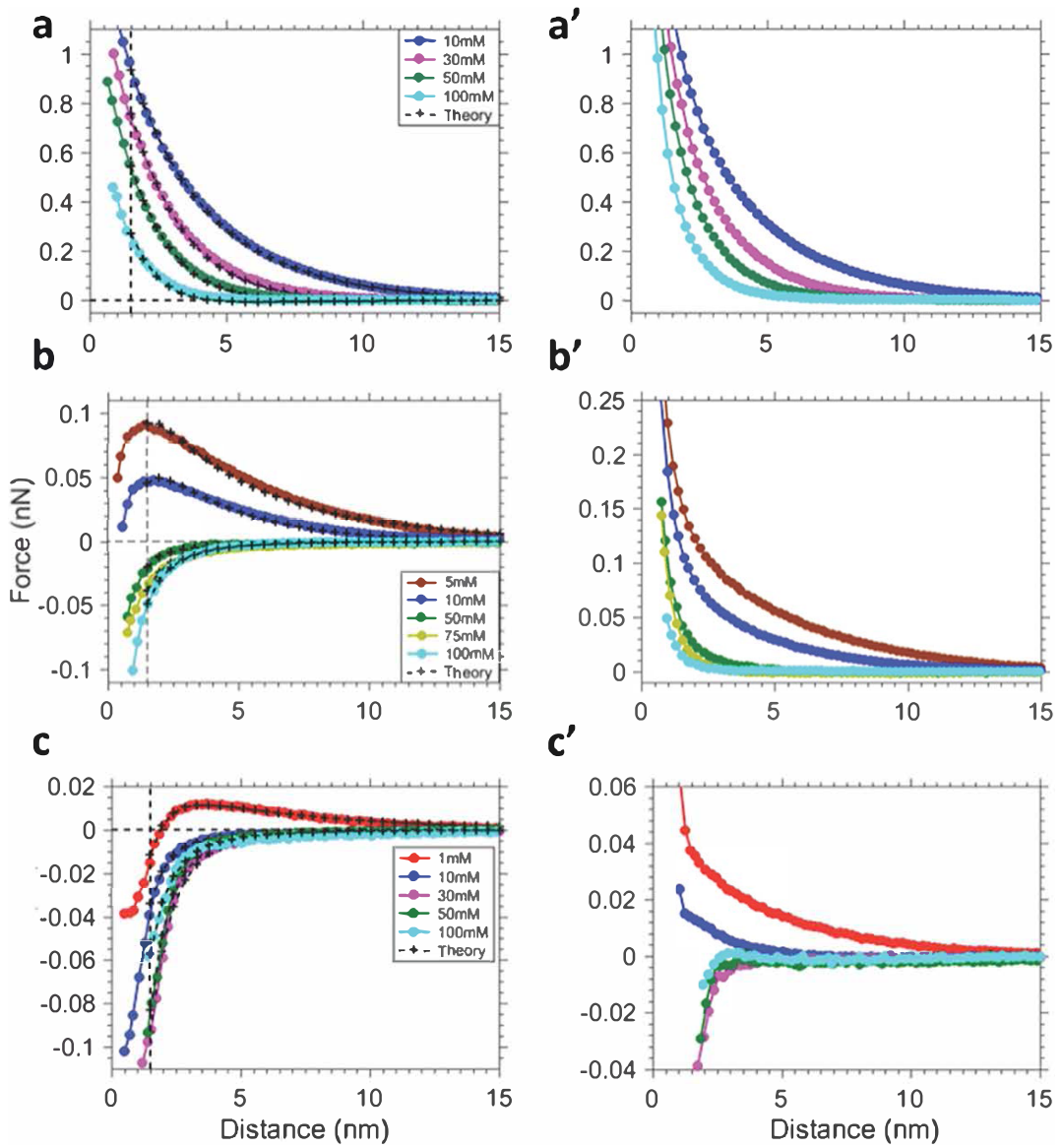

Figure 4. Tip-sample interaction force measurements using the AM-AFM mode on mica with (a) $\mathrm{NaCl},(\mathrm{b}) \mathrm{CsCl}$, and (c) $\mathrm{MgCl}_{2}$ salt solutions with variable concentrations. The black dotted curves in (a), (b), and (c) show the theoretically calculated force using DLVO theory $\left(a^{\prime}\right),\left(b^{\prime}\right)$, and $\left(c^{\prime}\right)$ show only the electrostatic part of the interaction force for the corresponding salt solutions. Note: Tip parameters used for $\mathrm{Na}^{+}$ions: $R=40 \pm$ $2 \mathrm{~nm}, k_{\mathrm{c}} \sim 1.25 \mathrm{~N} / \mathrm{m}, f_{0} \sim 29.9 \mathrm{kHz}$, and $Q \sim 3.5$ and for $\mathrm{Cs}^{+}$and $\mathrm{Mg}^{2+}: R=20 \pm 2 \mathrm{~nm}, k_{\mathrm{c}} \sim 0.463 \mathrm{~N} / \mathrm{m}, f_{0} \sim 17 \mathrm{kHz}, Q \sim 2.4$.

wetting with a contact angle determined by the value of $\Phi\left(h_{0}\right)$. On the basis of this scenario of ion adsorption-induced charge reversal, we could explain the transition from complete to partial wetting in our previous study that was limited to $\mathrm{Na}^{+}$, $\mathrm{K}^{+}, \mathrm{Ca}^{2+}$, and $\mathrm{Mg}^{2+}$ ions. ${ }^{54}$

AFM Investigation of Ion Adsorption. To test whether the mechanism described above also explains the alteration of the contact angles for all the salts in our present Hofmeister series of wettability, we determined the surface charge of mica-electrolyte interfaces for several types of salts using AFM. Solutions of $\mathrm{LiCl}, \mathrm{NaCl}, \mathrm{CsCl}, \mathrm{Mg}^{2+}$, and $\mathrm{Ca}^{2+}$ were analyzed at a fixed $\mathrm{pH}$ of 6 for salt concentrations from 1 to $100 \mathrm{mM}$. (For higher concentrations, efficient screening leads to very small forces and unreliable measurements, similar to streaming potential measurements.) Figure 4 shows the typical force curves for $\mathrm{NaCl}, \mathrm{CsCl}$, and $\mathrm{MgCl}_{2}$. While the total forces (left column) remain repulsive for $\mathrm{NaCl}$ for all salt concentrations, a transition to attractive forces was observed for both $\mathrm{CsCl}$ and $\mathrm{MgCl}_{2}$ for elevated concentrations at short distances. To reveal the contribution of the electrostatic forces, we first subtract the van der Waals contribution to the force following the procedures described in ref 56. Subsequently, we focus on the range of tip-sample separations of $\approx 2 \mathrm{~nm}$ and larger, for which short-range hydration forces are negligible. Doing so, we found that the electrostatic force is obviously repulsive for all concentrations of $\mathrm{NaCl}$. Yet, also for $\mathrm{CsCl}$ a small repulsive force prevails all the way up to the highest concentration investigated (Figure 4, right column). Only in the case of $\mathrm{MgCl}_{2}$, a clear reversal towards attractive electrostatic forces is observed at short distances beyond a threshold concentration of $\approx 10-30 \mathrm{mM}$. The latter implies a reversal of the surface charge consistent with our earlier streaming potential measurements. ${ }^{54}$ A similar behavior was found for $\mathrm{CaCl}_{2}$ solutions. In contrast, $\mathrm{LiCl}$ solutions were found to display very similar behavior as $\mathrm{NaCl}$, despite the fact that they do induce partial wetting at higher concentrations.

Using the procedures described in refs 56, 57, we converted these forces into surface charge densities, Figure 5. As explained in the Experimental section, the charge density probed by such a colloidal scale AFM spectroscopy measurement is the negative of the charge density of the diffuse part of the electric double layer, i.e., the sum of the intrinsic charge density of the mica surface and any counter ion (including protons) adsorbed in the Stern layer, extrapolated to infinite tip-sample separation. For the monovalent ions $\mathrm{Li}^{+}, \mathrm{Na}^{+}$, and $\mathrm{Cs}^{+}$, this surface charge is negative for all concentrations investigated. (Note, that the diffuse layer charge probed by the AFM is only a fraction of this intrinsic charge density of mica $\left(\approx 2 \mathrm{e} / \mathrm{nm}^{2}\right)$.) As anticipated based on the force curves, the magnitude of the surface charge decreases with increasing salt concentration for all monovalent cations. While it remains clearly negative for $\mathrm{Na}^{+}$and $\mathrm{Li}^{+}$, it approaches 0 within the error for $\mathrm{Cs}^{+}$for concentrations approaching $100 \mathrm{mM}$. In contrast, for the divalent cations, a clear reversal of the surface 


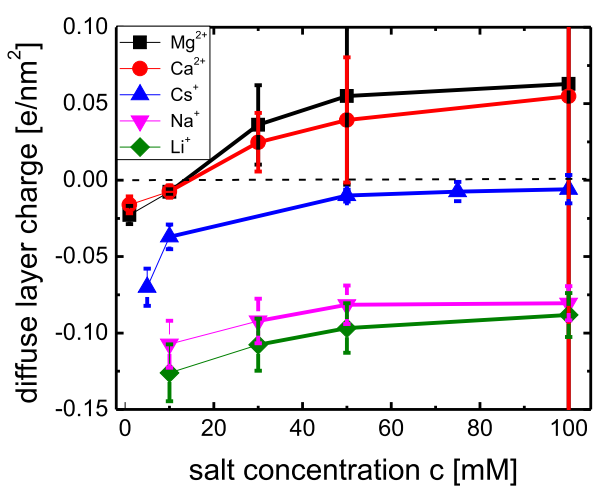

Figure 5. Diffuse layer charge $(\sigma)$ at the mica-electrolyte interface as determined by AFM spectroscopy: $\mathrm{LiCl}$ (green), $\mathrm{NaCl}$ (magenta), $\mathrm{CsCl}$ (blue), $\mathrm{CaCl}_{2}$ (red), and $\mathrm{MgCl}_{2}$ (black). The symbols are experimental datapoints based on 10-15 independent AFM experiments under identical conditions. The straight lines are guide to the eye.

charge is observed for concentrations of 10-20 mM, consistent with various reports in the literature. ${ }^{20,63}$ The larger error bars at high salt concentrations arise from the very small forces under these conditions due to efficient screening within the fitting range of $1.5-15 \mathrm{~nm}$.

The AFM measurements confirm the reversal of the surface charge for $\mathrm{Ca}^{2+}$ and $\mathrm{Mg}^{2+}$ that was reported earlier. For these systems, charge reversal indeed occurs in the same concentration range as the finite contact angles. This is consistent with our earlier claim that the wetting transition in these systems is caused by ion adsorption-induced charge reversal. For $\mathrm{Li}^{+}$and $\mathrm{Cs}^{+}$, however, we do not find charge reversal, despite the occurrence of finite contact angles. Unfortunately, the evidence is not completely conclusive given the increasing uncertainties of the AFM method for increasing concentrations. For $\mathrm{CsCl}$, the measurement suggests that the surface charge vanishes at the highest concentrations. Yet, the error bar includes the possibility of a small positive surface charge. For $\mathrm{LiCl}$, the surface charge remains almost constant at a negative value around $-0.1 \mathrm{e} / \mathrm{nm}^{2}$ up to $c=100$ $\mathrm{mM}$. Yet, the error bars at the highest concentration become very large-and the finite contact angles are only observed for even higher concentrations. Nevertheless, considering the AFM results as well as other classical reports in the literature, ${ }^{64}$ it seems highly unlikely that $\mathrm{Li}^{+}$ions and (to a somewhat lesser extent) $\mathrm{Cs}^{+}$ions induce charge reversal at mica-water interfaces at $\mathrm{pH}$ 6. Hence, it is also unlikely that our previous assignment of charge reversal as a driving force for the transition towards finite contact angles in the presence of divalent cations also holds for the monovalent ones. Instead, we would like to argue that the last term in eq 5, the contribution of hydration and short-range chemical effects, might play a decisive role. As already pointed out in refs 54, 55, this term plays an important role in the calculation of the exact location of the minimum of $\Phi(h)$ including the equilibrium contact angle. From our earlier ellipsometry measurements, we know that the equilibrium thickness $h_{0}$ of the wetting film frequently falls in the range below $2 \mathrm{~nm}$ for conditions of partial wetting. This is precisely the range for which hydration effects are important. ${ }^{9,10,64,65}$ Hydration effects arise from the complex interaction of water molecules with the mica surface and the ambient oil that are both modified by the presence of potentially adsorbed ions. In the first place, the presence of the interfaces gives rise to positional ordering of the water molecules. For atomically flat hydrophilic surfaces such as mica, this gives rise to a few discrete layers of water. ${ }^{66}$ For hydrophobic interfaces such as the water-oil interface, the water density is believed to decrease gradually within a few angstroms of the surface. In addition to the positional ordering, the hydrogen bonding network of water is affected by the (in)ability of forming $\mathrm{H}$-bonds with the surface. This effect in combination with local electric fields at the interface gives rise to an additional orientational ordering in the interfacial water. Both types of order typically have a range of not more than a few molecular diameters, i.e., typically $1-2 \mathrm{~nm}$, precisely the expected thickness of the wetting film next to the macroscopic drops. The contribution of hydration forces to the interface potential can be qualitatively understood in terms of the mean field model by Marčelja and Radic. ${ }^{60}$ According to this model, the mica-water and the water-oil interface interact via the order that they induce in the thin water film. This order is described by an order parameter $\eta$. $\eta$ vanishes in the bulk, but assumes finite values at the interfaces in response to local surface fields that-say-orient the water molecules. For the present system, the hydrophilic mica surface and the hydrophobic oil-water interface will impose rather different boundary conditions and order in the thin film. Microscopically, these boundary conditions arise for instance from the local electric fields at the interface that tend to orient the water molecules. The adsorption of cations to hydrophilic surfaces such as mica has a strong effect on the structure of the interfacial water, as shown in various recent X-ray reflectivity experiments $^{27}$ and numerical simulations. ${ }^{29,67}$ Our proposed scenario to explain the observed wetting transition implies that such ion adsorption-induced variations of the water structure give rise to an increased attractive interaction between the mica surface and the oil-water interface. In the case of $\mathrm{Rb}^{+}$and $\mathrm{Cs}^{+}$ ions, which are known to be poorly hydrated, ${ }^{68}$ interfacial adsorption would effectively "hydrophobize" the mica surface and thereby destabilize the thin water layer. For the case of $\mathrm{Li}^{+}$, however, this explanation would not apply. A detailed and complete understanding of all observations can therefore not be provided. Possibly, the difficulties to explain the phenomenon also illustrate the intrinsic deficiencies of extending continuum physics models all the way down to molecular scales where very specific chemical interactions are at play.

\section{CONCLUSIONS}

We investigated the dependence of the wettability of water on mica in ambient alkanes on the presence of dissolved chloride salts of a variety of alkali and earth alkaline cations. We observed a transition from near-zero to finite values of the contact angle (up to $\sim 15^{\circ}$ ). The ability of cations to induce partial wetting increases from $\mathrm{Na}^{+}, \mathrm{K}^{+}$to $\mathrm{Li}^{+}, \mathrm{Rb}^{+}, \mathrm{Cs}^{+}$and then to divalent cations $\left(\mathrm{Ca}^{2+}, \mathrm{Mg}^{2+}, \mathrm{Ba}^{2+}\right)$. We denote this ranking as cationic Hofmeister series of wettability alterationnotwithstanding the fact that the order of the ions differs from the conventional Hofmeister series related to the precipitation of cations. While the strong effect of the divalent cations stems from their strong adsorption to the mica surface and the resulting charge reversal of the interface, the occurrence of partial wetting in the case of the monovalent cations is tentatively attributed to the alteration of the hydration structure of the mica-electrolyte interface. We emphasize that the present cationic series applies to the specific mica/ 
water/alkane system. Nevertheless, given the fact that the negative surface charge of oil-water interfaces is rather common and robust, we expect that the mechanism of an ion adsorption-induced charge reversal should apply to a wider class of mineral surfaces. Elucidating that the role of hydration forces is a more complex challenge that will require more targeted experimental and numerical approaches than the measurements presented here.

\section{ASSOCIATED CONTENT}

\section{S Supporting Information}

The Supporting Information is available free of charge on the ACS Publications website at DOI: 10.1021/acs.langmuir.8b03297.

Precision in contact angle goniometry measurements; interfacial tension of representative monovalent and divalent salts at various concentrations; $\mathrm{CaCl}_{2}$ and $\mathrm{MgCl}_{2}$ contact angles on mica at all $\mathrm{pH}$ values and various concentrations; comparison of anions $\left(\mathrm{Cl}^{-}\right.$and $\mathrm{NO}_{3}{ }^{-}$) as the contributing factor in contact angles; hydrated ion radii of the cations used in our experiments (PDF)

\section{AUTHOR INFORMATION}

\section{Corresponding Author}

*E-mail: f.mugele@utwente.nl.

\section{ORCID}

\section{B. Bera: 0000-0002-8626-3682}

\section{Author Contributions}

${ }^{\dagger}$ B.B. and N.K. contributed equally to this work.

\section{Notes}

The authors declare no competing financial interest.

\section{ACKNOWLEDGMENTS}

The authors would like to thank BP Exploration Plc. for financial support for this work through the ExploRe program.

\section{REFERENCES}

(1) Dias, R.; Martinas, A. A.; Lima, R.; Mata, T. M. Single and TwoPhase Flows on Chemical and Biomedical Engineering; Bentham Science Publishers, 2012.

(2) Bibette, J.; Leal-Calderon, F.; Schmitt, V.; Poulin, P. Emulsion Science: Basic Principles. An Overview; Springer: Berlin, 2003.

(3) Lake, L. W. Enhanced Oil Recovery; Prentice Hall, 1989.

(4) Li, Y. Oil Recovery by Low Salinity Water Injection into a Reservoir: A New Study of Tertiary Oil Recovery Mechanism. Transp. Porous Media 2011, 90, 333-362.

(5) Muggeridge, A.; Cockin, A.; Webb, K.; Frampton, H.; Collins, I.; Moulds, T.; Salino, P. Recovery rates, enhanced oil recovery and technological limits. Philos. Trans. R. Soc., A 2014, 372, No. 20120320.

(6) Cacace, M. G.; Landau, E. M.; Ramsden, J. J. The Hofmeister series: salt and solvent effects on interfacial phenomena. Q. Rev. Biophys. 1997, 30, 241-277.

(7) Kunz, W. Specific Ion Effects, 1st ed.; World Scientific: Singapore, 2010.

(8) Kunz, W. Specific ion effects in colloidal and biological systems. Curr. Opin. Colloid Interface Sci. 2010, 15, 34-39.

(9) Butt, H. J.; Graf, K.; Kappl, M. Physics and Chemistry of Interfaces; Physics Textbook; Wiley: Weinheim, 2003.

(10) Israelachvili, J. N. Intermolecular and Surface Forces, 3rd ed.; Israelachvili, J. N., Ed.; Academic Press: San Diego, 2011; pp 71-90.

(11) Bruus, H. Theoretical Microfluidics; Oxford Master Series in Physics; OUP: Oxford, 2008.
(12) de Gennes, P. Wetting statics and dynamics. Rev. Mod. Phys. 1985, 57, No. 827.

(13) Lyklema, J. Fundamentals of Interface and Collloid Science, Volume III: Liquid-Fluid Interfaces; Academic Press: London, 2000.

(14) Onsager, L.; Samaras, N. N. T. The Surface Tension of Debye Huckel Electrolytes. J. Chem. Phys. 1934, 2, 528-536.

(15) dos Santos, A. P.; Diehl, A.; Levin, Y. Surface Tensions, Surface Potentials, and the Hofmeister Series of Electrolyte Solutions. Langmuir 2010, 26, 10778-10783.

(16) Ghosal, S.; Hemminger, J. C.; Bluhm, H.; Mun, B. S.; Hebenstreit, E. L. D.; Ketteler, G.; Ogletree, D. F.; Requejo, F. G.; Salmeron, M. Electron Spectroscopy of Aqueous Solution Interfaces Reveals Surface Enhancement of Halides. Science 2005, 307, 563566.

(17) Otten, D. E.; Shaffer, P. R.; Geissler, P. L.; Saykally, R. J. Elucidating the mechanism of selective ion adsorption to the liquid water surface. Proc. Natl. Acad. Sci. U.S.A. 2012, 109, 701-705.

(18) Behrens, S. H.; Borkovec, M. Electrostatic Interaction of Colloidal Surfaces with Variable Charge. J. Phys. Chem. B 1999, 103, 2918-2928.

(19) Pashley, R. M.; Israelachvili, J. N. Dlvo and hydration forces between mica surfaces in $\mathrm{Mg}^{2+}, \mathrm{Ca}^{2+}, \mathrm{Sr}^{2+}$, and $\mathrm{Ba}^{2+}$ chloride solutions. J. Colloid Interface Sci. 1984, 97, 446-455.

(20) Kékicheff, P.; Marcelja, S.; Senden, T.; Shubin, V. Charge reversal seen in electrical double layer interaction of surfaces immersed in 2:1 calcium electrolyte. J. Chem. Phys. 1993, 99, 6098.

(21) Scales, P. J.; Greaser, F.; Healy, T. Electrokinetics of the muscovite mica-aqueous solution interface. Langmuir 1990, 6, 582.

(22) Loh, S.-H.; Jarvis, S. P. Visualization of Ion Distribution at the Mica Electrolyte Interface. Langmuir 2010, 26, 9176-9178.

(23) Ricci, M.; Spijker, P.; Voïtchovsky, K. Water-induced correlation between single ions imaged at the solid liquid interface. Nat. Commun. 2014, 5, No. 4400.

(24) Fukuma, T.; Kobayashi, K.; Matsushige, K.; Yamada, H. True atomic resolution in liquid by frequency-modulation atomic force microscopy. Appl. Phys. Lett. 2005, 87, No. 034101.

(25) Siretanu, I.; Ebeling, D.; Andersson, M. P.; Stipp, S. L. S.; Philipse, A.; Stuart, M. C.; van den Ende, D.; Mugele, F. Direct observation of ionic structure at solid-liquid interfaces: a deep look into the Stern Layer. Sci. Rep. 2014, 4, No. 4956.

(26) Dishon, M.; Zohar, O.; Sivan, U. From Repulsion to Attraction and Back to Repulsion: The Effect of $\mathrm{NaCl}, \mathrm{KCl}$, and $\mathrm{CsCl}$ on the Force between Silica Surfaces in Aqueous Solution. Langmuir 2009, $25,2831-2836$.

(27) Lee, S. S.; Fenter, P.; Park, C.; Sturchio, N. C.; Nagy, K. L. Hydrated Cation Speciation at the Muscovite (001)-Water Interface. Langmuir 2010, 26, 16647-16651.

(28) Fukuma, T.; Reischl, B.; Kobayashi, N.; Spijker, P.; Canova, F. F.; Miyazawa, K.; Foster, A. S. Mechanism of atomic force microscopy imaging of three-dimensional hydration structures at a solid-liquid interface. Phys. Rev. B 2015, 92, No. 155412.

(29) Bourg, I. C.; Lee, S. S.; Fenter, P.; Tournassat, C. Stern Layer Structure and Energetics at Mica-Water Interfaces. J. Phys. Chem. C 2017, 121, 9402-9412.

(30) Lee, S. S.; Fenter, P.; Nagy, K. L.; Sturchio, N. C. Changes in adsorption free energy and speciation during competitive adsorption between monovalent cations at the muscovite (001)-water interface. Geochim. Cosmochim. Acta 2013, 123, 416-426.

(31) Pintea, S.; de Poel, W.; de Jong, A. E. F.; Vonk, V.; van der Asdonk, P.; Drnec, J.; Balmes, O.; Isern, H.; Dufrane, T.; Felici, R.; Vlieg, E. Solid-Liquid Interface Structure of Muscovite Mica in CsCl and $\mathrm{RbBr}$ Solutions. Langmuir 2016, 32, 12955-12965.

(32) Lyklema, J. Simple Hofmeister series. Chem. Phys. Lett. 2009, 467, 217-222.

(33) Lyklema, J. Lyotropic sequences in colloid stability revisited. Adv. Colloid Interface Sci. 2003, 100-102, 1-12.

(34) Parsons, D. F.; Ninham, B. W. Importance of Accurate Dynamic Polarizabilities for the Ionic Dispersion Interactions of Alkali Halides. Langmuir 2010, 26, 1816-1823. 
(35) Parsons, D. F.; Ninham, B. W. Charge Reversal of Surfaces in Divalent Electrolytes: The Role of Ionic Dispersion Interactions. Langmuir 2010, 26, 6430-6436.

(36) Parsegian, V. A.; Zemb, T. Hydration forces: Observations, explanations, expectations, questions. Curr. Opin. Colloid Interface Sci. 2011, 16, 618-624.

(37) Ball, P. Water as an Active Constituent in Cell Biology. Chem. Rev. 2008, 108, 74-108.

(38) Horinek, D.; Herz, A.; Vrbka, L.; Sedlmeier, F.; Mamatkulov, S. I.; Netz, R. R. Specific ion adsorption at the air/water interface: The role of hydrophobic solvation. Chem. Phys. Lett. 2009, 479, 173-183.

(39) Schwierz, N.; Horinek, D.; Netz, R. R. Reversed Anionic Hofmeister Series: The Interplay of Surface Charge and Surface Polarity. Langmuir 2010, 26, 7370-7379.

(40) Schwierz, N.; Horinek, D.; Netz, R. R. Anionic and Cationic Hofmeister Effects on Hydrophobic and Hydrophilic Surfaces. Langmuir 2013, 29, 2602-2614.

(41) Schwierz, N.; Netz, R. R. Effective Interaction between Two Ion-Adsorbing Plates: Hofmeister Series and Salting-In/Salting-Out Phase Diagrams from a Global Mean-Field Analysis. Langmuir 2012, $28,3881-3886$.

(42) Hofmeister, F. Zur Lehre von der Wirkung der Salze. Arch. Exp. Pathol. Pharmakol. 1888, 24, 247-260.

(43) Freundlich, H. Kapillarchemie, 2nd ed.; Akademische Verlagsgesellschaft: Leipzig, 1909.

(44) Zhang, Y.; Cremer, P. S. Chemistry of Hofmeister Anions and Osmolytes. Annu. Rev. Phys. Chem. 2010, 61, 63-83.

(45) Weissenborn, P. K.; Pugh, R. J. Surface Tension of Aqueous Solutions of Electrolytes: Relationship with Ion Hydration, Oxygen Solubility, and Bubble Coalescence. J. Colloid Interface Sci. 1996, 184, $550-563$.

(46) Boström, M.; Kunz, W.; Ninham, B. W. Hofmeister Effects in Surface Tension of Aqueous Electrolyte Solution. Langmuir 2005, 21, 2619-2623.

(47) Boström, M.; Williams, D. R. M.; Ninham, B. W. Ion Specificity of Micelles Explained by Ionic Dispersion Forces. Langmuir 2002, 18, 6010-6014.

(48) Kunz, W.; Lo Nostro, P.; Ninham, B. W. The present state of affairs with Hofmeister effects. Curr. Opin. Colloid Interface Sci. 2004, 9, 1-18.

(49) Boström, M.; Williams, D. R. M.; Ninham, B. W. Specific Ion Effects: Why DLVO Theory Fails for Biology and Colloid Systems. Phys. Rev. Lett. 2001, 87, No. 168103.

(50) Ben-Yaakov, D.; Andelmann, D. Revisiting the Poisson Boltzmann theory: Charge surfaces, multivalent ions and inter-plate forces. Phys. A 2010, 389, 2956.

(51) Sedev, R.; Fabretto, M.; Ralston, J. Wettability and surface energetics of rough fluoropolymer surfaces. J. Adhes. 2004, 80, 497520.

(52) Kondiparty, K.; Nikolov, A.; Wu, S.; Wasan, D. Wetting and Spreading of Nanofluids on Solid Surfaces Driven by the Structural Disjoining Pressure: Statics Analysis and Experiments. Langmuir 2011, 27, 3324-3335.

(53) Koopal, L. K. Wetting of Solid Surfaces: Fundamentals and Charge effects. Adv. Colloid Interface Sci. 2012, 179-182, 29-42.

(54) Mugele, F.; Bera, B.; Cavalli, A.; Siretanu, I.; Maestro, A.; Duits, M.; Cohen-Stuart, M.; Van Den Ende, D.; Stocker, I.; Collins, I. Ion adsorption-induced wetting transition in oil-water-mineral systems. Sci. Rep. 2015, 5, No. 10519.

(55) Cavalli, A.; Bera, B.; van den Ende, D.; Mugele, F. Analytic model for the electrowetting properties of oil-water-solid systems. Phys. Rev. E 2016, 93, No. 042606.

(56) Ebeling, D.; van den Ende, D.; Mugele, F. Electrostatic interaction forces in aqueous salt solutions of variable concentration and valency. Nanotechnology 2011, 22, No. 305706.

(57) Zhao, C.; Ebeling, D.; Siretanu, I.; van den Ende, D.; Mugele, F. Extracting local surface charges and charge regulation behavior from atomic force microscopy measurements at heterogeneous solidelectrolyte interfaces. Nanoscale 2015, 7, 16298-16311.
(58) Goldberg, R.; Chai, L.; Perkin, S.; Kampf, N.; Klein, J. Breakdown of hydration repulsion between charged surfaces in aqueous Cs+ solutions. Phys. Chem. Chem. Phys. 2008, 10, 49394945.

(59) Hachisu, S.; Kobayashi, Y. Kirkwood-Alder transition in monodisperse latexes. II Aqueous latexes of high electrolyte concentration. J. Colloid Interface Sci. 1974, 46, 470-476.

(60) Marčelja, S.; Radic, N. Repulsion of interfaces due to boundary water. Chem. Phys. Lett. 1976, 42, 129-130.

(61) Carnie, S. L.; Chan, D. Y. C. Interaction Free Energy between Plates with Charge Regulation: A Linearized Model. J. Colloid Interface Sci. 1993, 161, 260-264.

(62) Behrens, S. H.; Borkovec, M. Exact Poisson-Boltzmann solution for the interaction of dissimilar charge-regulating surfaces. Phys. Rev. E 1999, 60, 7040-7048.

(63) Baimpos, T.; Shrestha, B. R.; Raman, S.; Valtiner, M. Effect of Interfacial Ion Structuring on Range and Magnitude of Electric Double Layer, Hydration, and Adhesive Interactions between Mica Surfaces in $0.053 \mathrm{M} \mathrm{Li}+$ and Cs+ Electrolyte Solutions. Langmuir 2014, 30, 4322-4332.

(64) Pashley, R. DLVO and hydration forces between mica surfaces in $\mathrm{Li}^{+}, \mathrm{Na}^{+}, \mathrm{K}^{+}$, and $\mathrm{Cs}^{+}$electrolyte solutions: A correlation of doublelayer and hydration forces with surface cation exchange properties. J. Colloid Interface Sci. 1981, 83, 531-546.

(65) Kanduč, M.; Schlaich, A.; Schneck, E.; Netz, R. R. Hydration repulsion between membranes and polar surfaces: Simulation approaches versus continuum theories. Adv. Colloid Interface Sci. 2014, 208, 142-152.

(66) Fukuma, T.; Ueda, Y.; Yoshioka, S.; Asakawa, H. Atomic-Scale Distribution of Water Molecules at the Mica-Water Interface Visualized by Three-Dimensional Scanning Force Microscopy. Phys. Rev. Lett. 2010, 104, No. 016101.

(67) Sakuma, H.; Kawamura, K. Structure and dynamics of water on $\mathrm{Li}^{+}-, \mathrm{Na}^{+}-, \mathrm{K}^{+}-, \mathrm{Cs}^{+}-, \mathrm{H}_{3} \mathrm{O}^{+}$- exchanged muscovite surfaces: A molecular dynamics study. Geochim. Cosmochim. Acta 2011, 75, 6381.

(68) Marcus, Y. Effect of Ions on the Structure of Water: Structure Making and Breaking. Chem. Rev. 2009, 109, 1346-1370. 